\title{
Modelo experimental de isquemia/reperfusão intestinal por clampeamento de aorta abdominal em ratos Wistar
}

\section{Experimental model of mesenteric ischemia/reperfusion by abdominal aorta clamping in Wistar rats}

\author{
Bruno da Costa Rocha'; Rogério Rafael da Silva Mendes²; Gabriel Varjão lima²; Gabriel de Souza Albuquerquez; Lucas Lacerda \\ Araújoz; Mateus Neves da Silva de Jesus²; Washington luís Conrado dos Santos ${ }^{3}$; Mário Castro Carreiro ${ }^{4}$
}

R E S U M O

\begin{abstract}
Objetivo: desenvolver um modelo experimental de isquemia global normotérmica transitória capaz de demonstrar os tempos de isquemia e reperfusão necessários para desenvolvimento de lesão de isquemia/reperfusão em intestinos delgados de ratos Wistar através clampeamento de aorta abdominal suprarrenal. Métodos: Vinte ratos Wistar adultos machos, pesando entre 250 e $350 \mathrm{~g}$, foram distribuídos aleatoriamente em cinco grupos, com quatro ratos cada, e submetidos a tempos crescentes de isquemia (0 - 30 - 45 - 60 - 90 minutos). Dentro de cada grupo, à exceção do grupo controle, dois ratos foram submetidos à 60 minutos de reperfusão e dois à 90 minutos. Após os procedimentos, procedeu-se análise histológica através de medição de áreas de necrose. Resultados: O grau de necrose intestinal variou de 15 a 54\% ( $p=0,0004)$. Houve tendência de aumento progressivo no grau de lesão relacionado ao aumento no tempo de isquemia, contudo, os maiores graus de lesão foram observados nos menores tempos de reperfusão. A análise do coeficiente de variação de necrose entre os dez grupos de isquemia/reperfusão demonstrou diferença estatisticamente significante em 15 postos, sendo 13 relacionados ao grupo controle. Conclusão: O modelo foi capaz de demonstrar os tempos necessários para que ocorra lesão de isquemia/reperfusão intestinal através de clampeamento aórtico e poderá servir como base para facilitar o desenvolvimento de estudos voltados para a compreensão deste tipo de lesão.
\end{abstract}

Descritores: Desenvolvimento experimental. Isquemia. Reperfusão. Constrição. Aorta abdominal. Intestino delgado.

INTRODUÇÃO

A pós um período crítico de isquemia, a restauração do fluxo sanguíneo em determinado órgão desencadeia o processo de lesão de isquemia/reperfusão (I/R $)^{1}$. Isso ocorre porque a isquemia cria condições para que, no momento da reperfusão, enzimas ativadas participem da redução do oxigênio molecular gerando oxirradicais ${ }^{2}$.

A lesão de I/R é um fenômeno que atrai o interesse de pesquisadores, os quais, através de modelos experimentais, buscam entender sua fisiopatologia e possíveis medidas terapêuticas. Observa-se, no entanto, o desenvolvimento majoritário de modelos de I/R regionais ${ }^{3-5}$, através de clampeamento seletivo de pedículos vasculares para órgãos específicos, em detrimento de modelos de I/R global de órgãos, através de clampeamento de vasos-fonte para vários órgãos. Nesse contexto, encontram-se os modelos de clampeamento de aorta, que teriam importância ao simular operações de aorta e suas repercussões sistêmicas, como comprometimentos neurológico, intestinal e renal ${ }^{6-8}$.

O objetivo deste estudo foi desenvolver um modelo experimental de isquemia global normotérmica transitória capaz de demonstrar os tempos de isquemia e reperfusão necessários para desenvolvimento de lesão de I/R em intestinos delgados de ratos Wistar através do clampeamento da aorta abdominal suprarrenal.

\section{MÉTODOS}

Foram utilizados 20 ratos Wistar adultos machos, pesando entre 250 e $350 \mathrm{~g}$, mantidos sob condições controladas, com água ad libitum e alimentados com ração comercial. Os procedimentos experimentais foram aprovados pelo Comitê de Ética da instituição. Número do protocolo 03/09.

Trabalho realizado no Núcleo de Pesquisas Experimentais, Departamento de Cirurgia Experimental e Especialidades Cirúrgicas, Faculdade de Medicina da Universidade Federal da Bahia (UFBA) em parceria com o Centro de Pesquisa Gonçalo Moniz, Laboratório de Patologia e Biointervenção (LPBI), Fiocruz.

1. Professor Substituto do Departamento de Cirurgia Experimental e Especialidades Cirúrgicas da Faculdade de Medicina da Bahia, FMB/UFBA 2. Médico e ex-estudante de Graduação de Medicina, UFBA, Bahia, Brasil; 3.Patologista Pesquisador do Laboratório de Patologia e Biointervenção (LPBI), Fiocruz, Salvador, Bahia, Brasil; 4. Professor Adjunto do Departamento de Cirurgia Experimental e Especialidades Cirúrgicas da Faculdade de Medicina da Bahia, FMB/UFBA. 
Os animais foram distribuídos aleatoriamente em cinco grupos (Tabela 1). Todos foram anestesiados com quetamina $(75 \mathrm{mg} / \mathrm{Kg})$ e xilazina $(10 \mathrm{mg} / \mathrm{Kg})$, administrados por via intramuscular. Foram mantidos em ventilação espontânea, posicionados na mesa cirúrgica em decúbito dorsal, imobilizados pelas quatro extremidades e submetidos à tricotomia abdominal e assepsia com povidine degermante. Foi realizada laparotomia xifopúbica por planos, com posterior exposição da cavidade abdominal. O rim esquerdo foi rebatido para a direita e a gordura perirrenal divulsionada. Após dissecção, a aorta abdominal foi clampeada logo acima da emergência da artéria renal direita com pinça hemostática atraumática (Figura 1), interrompendo o fluxo sanguíneo mesentérico.

Após completar os tempos de isquemia e reperfusão, foram realizadas secção de veia cava inferior e ressecção em bloco dos órgãos abdominais. Nos animais do Grupo I (controle), logo após a laparotomia era realizado esse procedimento. O intestino delgado foi colocado em solução de formalina ácida por 24 horas e depois mantidos em álcool $70 \%$ até a análise histopatológica. Para cada rato, foram obtidas seis secções de intestino delgado, das quais duas foram aleatoriamente escolhidas para análise histopatológica após fotografias das paredes intestinais. Todas as imagens foram obtidas com resolução de 1280x1024 pixels, usando uma câmera de captura (CX41, Olympus, Tóquio, Japão) acoplada ao microscópio óptico com lentes de aumento 20x e analisadas com auxílio do software ImageJ (National Institute of Health, Bethesda, Estados Unidos). A análise das imagens ocorreu através de medição de áreas de necrose em relação à área total das paredes intestinais nas secções. Três pesquisadores cegos para os grupos dos ratos realizaram as medições. A média das áreas medidas por cada um dos pesquisadores foi considerada para análise dos resultados.

A análise estatística descritiva e inferencial foi realizada utilizando o teste não-paramétrico de KruskalWallis e, quando aplicável, o teste de Student-NewmanKeuls. Foi assumido como estatisticamente significativo o valor $\mathrm{p}<0,05$.

\section{RESULTADOS}

O grau de lesão intestinal, em necrose, foi avaliado nos 20 ratos submetidos a diferentes tempos de isquemia e reperfusão (Tabela 1). Os valores médios de grau de necrose intestinal variaram entre 15\% e 54\% (Figura 2).

Foi observada diferença estatisticamente significativa ( $p=0,0004$ ) entre os graus de necrose nos grupos estudados, com tendência de aumento progressivo relacionada ao aumento dos tempos de isquemia ( 0 - $30-45-$ 60 - 90 minutos). Contudo, os graus de necrose foram maiores nos menores tempos de reperfusão (60 minutos) em comparação com os grupos de maior tempo (90 minu-

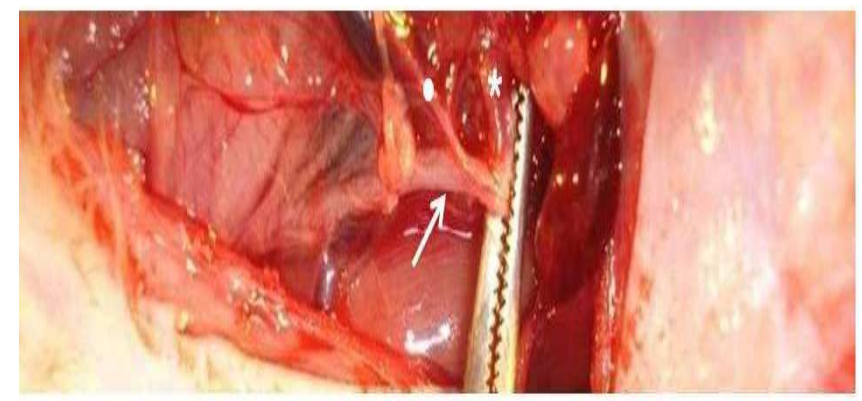

Figura 1 - Artéria renal esquerda rebatida (•); aorta abdominal (seta) clampeada logo acima da emergência da artéria renal direita (*).

Tabela 1 - Desenho do estudo.

\begin{tabular}{|c|c|c|c|}
\hline Grupo & $\begin{array}{l}\text { Isquemia } \\
\text { (minutos) }\end{array}$ & $\begin{array}{c}\text { Reperfusão } \\
\text { (minutos) }\end{array}$ & $\mathrm{n}$ \\
\hline I & 0 & 0 & 4 \\
\hline II & 30 & & 4 \\
\hline a & & 60 & 2 \\
\hline$b$ & & 90 & 2 \\
\hline III & 45 & 4 & \\
\hline a & & 60 & 2 \\
\hline $\mathrm{b}$ & & 90 & 2 \\
\hline IV & 60 & 4 & \\
\hline a & & 60 & 2 \\
\hline $\mathrm{b}$ & & 90 & 2 \\
\hline V & 90 & 4 & \\
\hline a & & 60 & 2 \\
\hline$b$ & & 90 & 2 \\
\hline
\end{tabular}

tos). Não foram encontradas diferenças estatisticamente significativas entre os diferentes tempos de reperfusão para um mesmo tempo de isquemia.

O grupo controle demonstrou ser o de menor grau de necrose em relação aos demais grupos estudados. Quando analisados individualmente os graus de necrose intergrupos, o coeficiente de variação demonstrou diferença entre as médias estatisticamente significativa em 15 dos 45 postos, sendo 13 implicados diretamente ao grupo controle (Tabela 2). Este grupo apresentou diferença estatisticamente significante quando comparado aos demais grupos de IR em $76 \%$ das vezes.

\section{DISCUSSÃO}

Em ratos, a emergência da artéria mesentérica superior localiza-se abaixo das emergências das artérias renais. Logo, o clampeamento da aorta acima das emergências das artérias renais implica cessação do fluxo mesentérico. 
Tabela 2 - Diferença entre as médias dos grupos de Isquemia/Reperfusão.

\begin{tabular}{lcc}
\hline Grupos & Coeficiente de variação & p-valor \\
\hline Controle x I45R60 & 29,3333 & 0,00 \\
Controle x I60R60 & 38,5 & 0,00 \\
Controle x I60R90 & 25,8333 & 0,01 \\
Controle x I90R60 & 30,5 & 0,00 \\
Controle x I90R90 & 23,5 & 0,02 \\
Controle x I30R60 & 22,3333 & 0,03 \\
Controle x I30R90 & 19,8333 & 0,05 \\
Controle x I45R60 & 32,6667 & 0,00 \\
Controle x I45R90 & 20,1667 & 0,05 \\
Controle x I60R60 & 41,8333 & $<0,0001$ \\
Controle x I60R90 & 29,1667 & 0,00 \\
Controle x I90R60 & 33,8333 & 0,00 \\
Controle x I90R90 & 26,8333 & 0,01 \\
I30R90 x I60R60 & 22 & 0,03 \\
I45R90 x I60R60 & 21,6667 & 0,03 \\
\hline
\end{tabular}

(p) Teste não paramétrico de Kruskal-Wallis; $p=0,0004$;

Posterior Student-Newman-Keuls test; $p<0,05=$ estatisticamente significativo

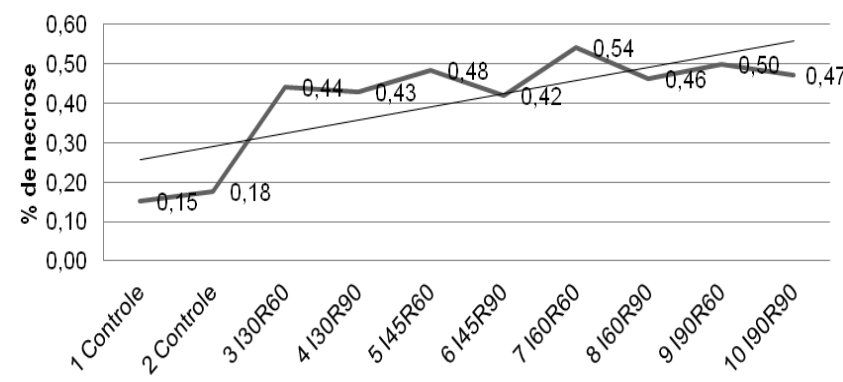

Figura 2 - Curva de isquemia/reperfusão.

A lesão tecidual isquêmica é um evento comum na prática cirúrgica. A restauração do fluxo sanguíneo, mesmo sendo crucial para evitar a morte celular, está associada ao agravamento do dano isquêmico por lesão de l/ $\mathrm{R}^{1}$. O clampeamento aórtico executado no reparo aberto de aneurismas da aorta está associado à alterações hemodinâmicas que acarretam lesão de I/R ${ }^{9}$. Estes danos já foram estudados através de diversos modelos experimentais baseados em pinçamento do pedículo vascular mesentérico 3,4,10.

Observa-se nas pesquisas já realizadas sobre o tema, que não há padronização dos tempos de isquemia e reperfusão essenciais para a ocorrência de lesões teciduais, fato já notado por Silveira et al. ${ }^{11}$. Visando melhor avaliar esta questão no intestino, distintos períodos foram testados de forma a analisar a graduação das alterações histológicas, bem como, a duração específica da isquemia e reperfusão necessária para o surgimento de lesões no órgão em questão.

O intestino delgado é um órgão que sabidamente sofre bastante com as lesões I/R. Desde que
Schoenberg e Berger ${ }^{12}$ definiram que, com uma hora de isquemia, a mucosa intestinal já possuía danos importantes e, com duas horas, os danos já seriam irreversíveis. Vários estudos foram desenvolvidos, geralmente com o tempo variando de uma a duas horas de isquemia. De acordo com Sola et al. ${ }^{13}$, após 90 minutos de isquemia e 30 minutos de reperfusão, por oclusão da artéria mesentérica, este órgão apresentou lesão histológica grau 5 , segundo a classificação modificada por Chiu et al. ${ }^{14}$. Santos et al..$^{15}$ demonstraram que, com 30 minutos de isquemia seguidos de 60 minutos de reperfusão, o grau das lesões histológicas obtidas variam entre 3 e 4 . Li et al. ${ }^{16}$ observaram que, 60 minutos isquemia e 60 minutos de reperfusão intestinal, a partir do pinçamento da ateria mesentérica superior, causam significativa lesão da mucosa. Brito et al. ${ }^{10}$ demonstraram que, para um mesmo tempo de isquemia, um maior grau de lesão está associado ao maior tempo de reperfusão. Miranda et al. ${ }^{17}$ utilizaram em seu estudo 45 minutos de isquemia seguidos de sete dias de reperfusão.

Nesse estudo, optou-se por realizar clampeamento de aorta. Os motivos para a escolha desse vaso foram: escassez de estudos com esse tipo de clampeamento e facilidade de manipulação do vaso. Com relação à isquemia, estes resultados são corroborados por dados da literatura que mostram que quanto maior o tempo de isquemia, maior o grau da lesão histológica ${ }^{18}$. Neste estudo validaram-se os tempos, mostrando que, a partir de 30 minutos de isquemia na aorta, já é possível notar alteração histológica na parede do intestino dos ratos. Não foram encontradas variações estatisticamente significativas entre as lesões nos tempos de reperfusão de 60 e 90 minutos em nenhum dos grupos. 
Em conclusão, o modelo foi capaz de demonstrar os tempos necessários para que ocorra lesão de l/R intestinal através de clampeamento aórtico e poderá servir como base para facilitar o desenvolvimento de estudos voltados para a compreensão deste tipo de lesão.

\section{Agradecimentos}

À Doutora Maria das Graças Farias Pinto, pelo auxílio com o cuidado com os animais, e à doutoranda Joselli Santos Silva, pelo auxílio na análise histológica.

\section{A B S T R A C T}

Objective: To develop an experimental model of global normothermic ischemia able to demonstrate the transient ischemia and reperfusion periods required for development of ischemia/reperfusion injury in the small intestines of Wistar rats by clamping the abdominal aorta. Methods: Twenty adult male Wistar rats weighing 250-350g were randomly divided into five groups with four rats each and submitted to increasing times of ischemia (0 - 30-45-60 - 90 minutes). Within each group, except the control one, two rats underwent 60 minutes of reperfusion and two 90 minutes. After the procedures, histological analysis was conducted by measurement of areas of necrosis. Results: The degree of intestinal necrosis ranged from $15 \%$ to $54 \%$ ( $p=0.0004)$. There was progressive increase in the degree of injury related to increase in ischemic time. However, greater degrees of injury were observed in the lowest times of reperfusion. The analysis of the coefficient of variation of necrosis among the ten groups of ischemia/ reperfusion showed a statistically significant difference in 15 areas, 13 related to the control group. Conclusion: The model was able to show the periods required for the occurrence of ischemia/reperfusion injury by aortic clamping and can serve as a basis to facilitate the development of studies that aim at understanding this kind of injury.

Key words: Wistar rats. Abdominal aorta. Small intestine. Ischemia. Reperfusion.

\section{REFERÊNCIAS}

1. Silva Júnior OC, Centurion S, Pacheco EG, Brisotti JL, Oliveira AF, Sasso KD. Aspectos básicos da lesão de isquemia e reperfusão e do pré-condicionamento isquêmico. Acta cir bras. 2002;17(supl.3):96-100.

2. Ferro CO, Chagas VLA, Oliveira MF, Oliveira PL, Schanaider A. Atividade da catalase no pulmão, rim e intestino delgado não isquemiado de ratos após reperfusão intestinal. Rev Col Bras Cir. 2010;37(1):31-8.

3. Uygun M, Yilmaz S, Pekdemir M, Duman C, Gürbüz YS. The diagnostic value of ischemia-modified albumin in a rat model of acute mesenteric ischemia. Acad Emerg Med. 2011;18(4):355-9.

4. Tang ZH, Qiang JW, Feng XY, Li RK, Sun RX, Ye XG. Acute mesenteric ischemia induced by ligation of porcine superior mesenteric vein: multidetector CT evaluations. Acad Radiol. 2010;17(9):1146-52.

5. Silva FN. Isquemia hepática normotérmica em ratos: estudo da lesão celular através do uso de clampeamento pedicular contínuo e intermitente. Rev Col Bras Cir. 2002;29(6):342-8.

6. Mônaco BA, Benício A, Contreras ISB, Mingrone LE, Ballester G, Moreira LFP. Pré-condicionamento Isquêmico e monitorização da função medular na abordagem da aorta torácica descendente. Arq bras cardiol. 2007;88(3):291-6.

7. Studer W, Wu X. Supraceliac aortic cross-clamping and declamping. Effects of dopexamine and dopamine on systemic and mesenteric hemodynamics, metabolism and intestinal tonometry in a rat model. Acta Anaesthesiol Scand. 2000;44(3)241-8.

8. Feitosa EAN, Taha MO, Fagundes DJ, Takiya CM, Cardoso LR, Campo DM. Estudo da morfologia renal após a oclusão da aorta abdominal infrarrenal em ratos. Rev Col Bras Cir. 2005;32(4):178-82.

9. Juel IS, Solligård E, Lyng $O$, Strømholm $T$, Tvedt KE, Johnsen $H$, et al. Intestinal injury after thoracic aortic cross-clamping in the pig. J Surg Res. 2004;117(2):283-95.

10. Brito MVH, Araújo M, Acácio GJS, Acácio GJS, Reis JMC. Lesão intestinal após isquemia-reperfusão: estudo comparativo usando sal tetrazólico (MTT) e histologia. Acta cir bras. 2001;16(1):26-31.

11. Silveira $M$, Yoshida WB. Isquemia e reperfusão em músculo esquelético: mecanismos de lesão e perspectiva de tratamento. J vasc bras. 2004;3(4):367-78.
12. Schoenberg MH, Berger HG. Reperfusion injury after intestinal ischemia. Crit Care Med. 1993;21(9):1376-86.

13. Sola A, Hotter G, Prats N, Xaus C, Gelpi E, Roselló-Catafau J. Modification of oxidative stress in response to intestinal preconditioning. Transplantation. 2000;69(5):767-72.

14. Chiu CJ, McArdle AH, Brown R, Scott HJ, Gurd FN. Intestinal mucosal lesion in low-flow states. I. A morphological, hemodynamic, and metabolic reappraisal. Arch Surg. 1970;101(4):478-83.

15. Santos CHM, Gomes OM, Pontes JCDV, Miiji LNO, Bispo MAF. The ischemic preconditioning and postconditioning effect on the intestinal mucosa of rats undergoing mesenteric ischemia/reperfusion procedure. Acta cir bras. 2008;23(1):22-8.

16. Li YS, Wang ZX, Li C, Xu M, Li Y, Huang WQ, et al. Proteomics of ischemia/reperfusion injury in rat intestine with and without ischemic postconditioning. J Surg Res. 2010;164(1):e173-80.

17. Miranda EF, Greca FH, Noronha L, Kotze LR, Rubin MR. A influência do azul de metileno na cicatrização de anastomoses intestinais submetidas à isquemia e reperfusão em ratos. Acta cir bras. 2010;25(1):63-70.

18. Guan Y, Worrell RT, Pritts TA, Montrose MH. Intestinal ischemiareperfusion injury: reversible and irreversible damage imaged in vivo. Am J Gastrointest Liver Physiol. 2009;297(1):G187-96.

Recebido em 03/09/2011

Aceito para publicação em 03/11/2011

Conflito de interesse: nenhum

Fonte de financiamento: nenhum

\section{Como citar este artigo:}

Mendes RRS, Lima GV, Albuquerque GS, Araújo LL, Jesus MNS, Santos WLC, Carreiro MC, Rocha BC. Modelo experimental de isquemia/ reperfusão intestinal por clampeamento de aorta abdominal em ratos wistar. Rev Col Bras Cir. [periódico na Internet] 2012; 39(3). Disponível em URL: http://www.scielo.br/rcbc

Endereço para correspondência:

Bruno da Costa Rocha

E-mail: brunorochaccv@hotmail.com 